\title{
A case report of recurrent syncope \& transient ischaemic attack in elderly man
}

Paul GK ${ }^{*}$ and Bari MS ${ }^{2}$

${ }^{1}$ Assistant Professor, Department of Cardiology, Mymensingh Medical College (MMC), Mymensingh, Bangladesh

${ }^{2}$ Professor \& Head of the Department of Cardiology, Mymensingh Medical College (MMC), Mymensingh, Bangladesh

\begin{abstract}
Cardiac myxomas may present clinically with different features. The most frequent initial neurological manifestation is TIA. Left atrial myxoma (LAM), the most common primary tumours of the heart are unusual or rare in the elderly. Moreover, elderly patients often present with non-specific symptoms that are frequently overlooked which makes an early diagnosis challenging. Left atrial myxoma (LAM), if left untreated, is inexorably progressive and usually fatal. It is an important source of central nervous system embolisom. We describe the case of an 85-year-old man with left atrial myxoma with Mitral regurgitation (Grade-1+) with Atrial fibrillation (AF) with Hypertension with left heart failure, having recurrent palpitation, pre-syncope and transient ischaemic attack and the diagnosis was suspected clinically to some extent but discovered primary etiology by trans-thoracic echocardiography (TTE). Due to low morbidity and mortality besides its excellent outcome even in the elderly, surgery is the preferred choice of treatment for LAMs.
\end{abstract}

\section{Introduction}

Intracardiac myxoma is the most frequent benign tumour of the heart. Although most (75\%) are located in the left atrium (LA), myxomas are also found in the right atrium (18\%), right ventricle (4\%), and left ventricle (3\%).They occur in all age groups, most frequently between third and sixth decade, while it is rare in the ninth decade $[1,2]$. Left atrial myxoma(LAM) is histologically benign and is the most common primary tumour of the heart [3]. It accounts for $80 \%$ of all cardiac tumours [4]. Differential diagnosis of atrial myxoma includes pedunculated thrombus, metastatic sarcoma and melanoma in left atrium [3]. Atrial myxoma in older people is considered to be unusual, but with the advent of newer imaging modalities and longer life expectancy, the incidence of myxoma in older has increased [1]. Atrial myxoma may be asymptomatic, in contrast, symptomatic cases may present with obstruction of cardiac blood flow, embolic phenomena and constitution symptoms. Common symptoms include chest pain, dyspnea, orthopnea, peripheral embolism or syncope [2,5,6]. Left atrial myxoma (LAM), if left untreated, is inexorably progressive and usually fatal. It is an important source of central nervous system embolisom and producing TIA as an initial neurological manifestation before producing cardiac symptom in some cases [7].

\section{Case presentation}

An 85 year old gentleman presented with a 3 week history of shortness of breath, recurrent palpitation, pre-syncope and syncope. He is smoker, hypertensive but non-diabetic. In the previous three years he had suffered from an episode of transient ischaemic attack (TIA), mild exertional shortness of breath (New York Heart Association/NYHA, class-2) and chest pain (Canadian Cardiovascular Society/CCS, Grade-2). He also notice gradual weight loss and generalized weakness. Yet now He maintains normal daily activities with taking some help. On examination, pulse is irregularly irregular but normal ventricular rate, blood pressure-140/90 $\mathrm{mmHg}$ with anti-hypertensive drugs. Patient having bilateral basal lung crepitation and found a loud and variable first heart sound (S1), diastolic murmur (Grade-2/6) in the mitral area. His ECG showed Atrial fibrillation (AF) with slow ventricular rate (90/minute) and left ventricular hypertrophy (LVH) (Figure 1). Chest X-ray revealed boarder line cardiomegaly and hypertensive heart (Figure 2). Echocardiogram showed a large size mobile mass (35 $\times 30 \mathrm{~mm}$ ) (Figure 3 ) in the dilated left atrium (LA) attached to the interatrial septum, MR (G-1+, degenerative) (Figure 4) with fair left ventricular (LV) systolic function (LVEF-50\% to 55\%). CT scan of the Brain showed unremarkable but some age related degenerative change

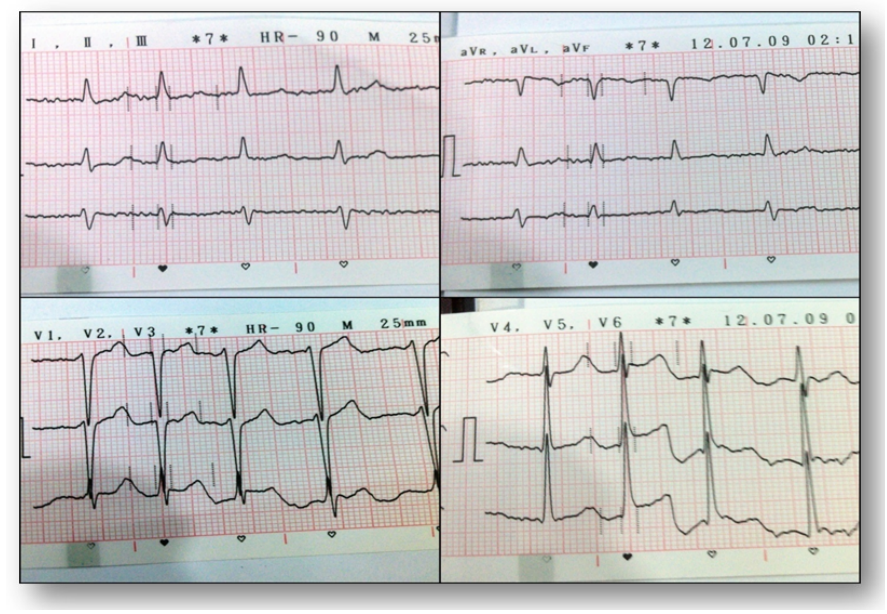

Figure 1. Electrocardiogram showing Atrial fibrillation \& LVH (Voltage criteria).

Correspondence to: Dr. Gobinda Kanti Paul, Assistant Professor, Department of Cardiology, Mymensingh Medical College (MMC), Mymensingh, Bangladesh; E-mail: drgobinda@yahoo.com

Key words: atrial myxoma, benign tumour, atrial fibrillation, TIA

Received: April 29, 2016; Accepted: May 25, 2016; Published: May 28, 2016 


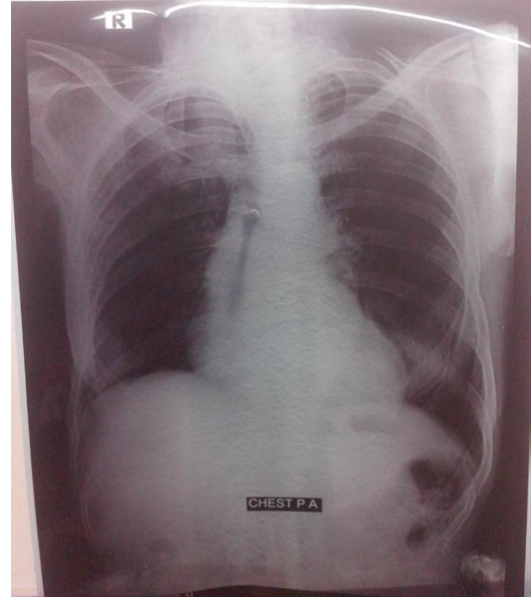

Figure 2. Chest X-ray showing Cardiac shadow boarder line enlarged \& long left boarder

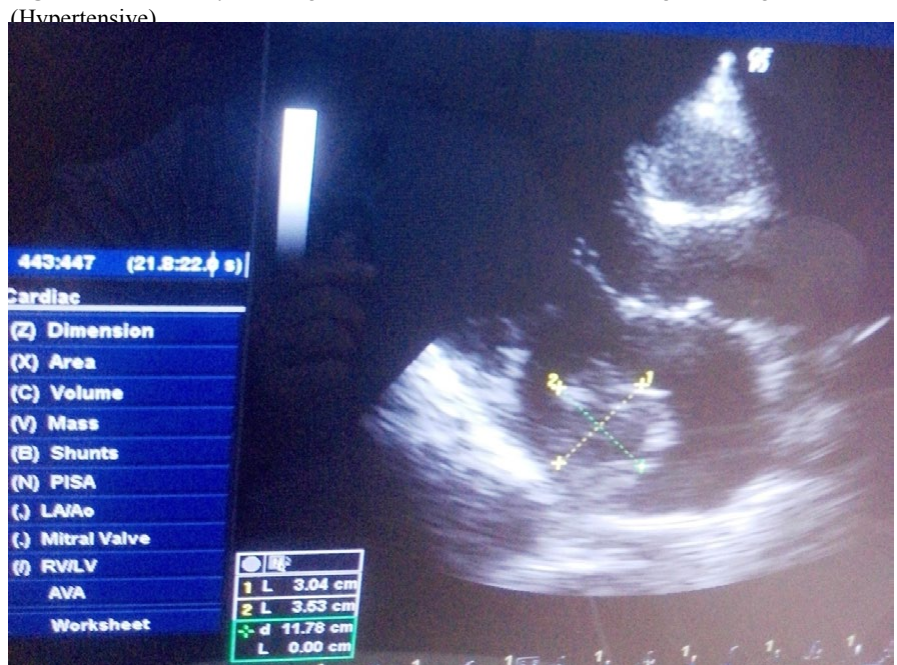

Figure 3. Echocardiogram showing a large mass size: $35 \times 30 \mathrm{~mm}$ in the dilated left atrium.

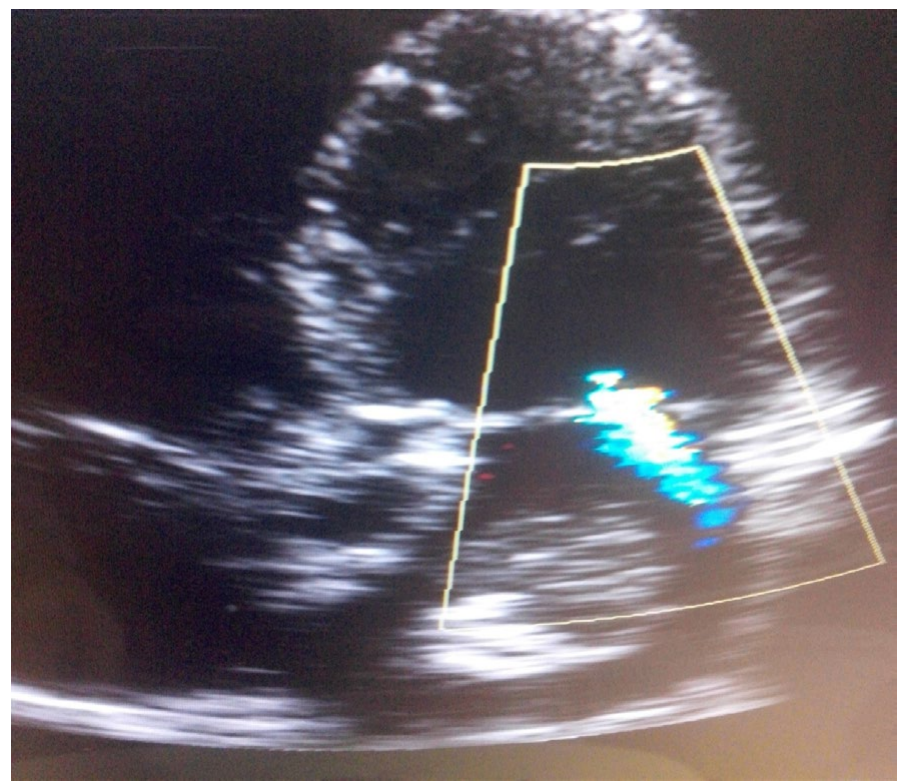

Figure 4. Echocardiogram 2D guided color flow mapping (CFM) showing Large Mass lesion in the dilated left atrium and also MR (G-1+, degenerative). in the brain. Others routine blood tests, patient is mildly anaemic (Hb$10 \mathrm{gm} / \mathrm{dl}$ ) but renal, liver function tests and also ultrasound of whole abdomen are normal. So our case is Left atrial myxoma with AF with Hypertension with Left heart failure. Now patient is clinically stable with adequate treatment and resume his ordinary daily activities with taking some help.

\section{Discussion}

When a mass inside the heart is diagnosed it usually represents a thrombus or vegetation. If a cardiac mass is indeed a tumour the most likely cause is a metastatic process from remote malignancy (usually from breast, Lung or malignant melanoma) [8].

Left atrial myxoma is most commonly seen in women with $90 \%$ being solitary and pedunculated and $10 \%$ being familial, with an autosomal dominant pattern of inheritance $[9,10]$. In $5 \%$ of cases myxomas can be multiple. Myxomas characteristically arise from or near the interatrial septum at the border of the fossa ovalis membrane. The diagnosis of atrial myxoma can be elusive, especially when symptoms are suggestive of other diagnoses. In this case, the significance of these patients past medical history of a transient ischemic attack only became apparent when the patient presented with new symptoms of AF, which led to various investigations looking for a source of cardiogenic cerebral embolism, eventually revealing the left atrial myxoma. Elderly patients often present with non specific symptoms that are often overlooked in the absence of a supporting cardiac history which makes an early diagnosis challenging [11]. Left atrial myxoma presenting in seventh decade is rare, with only few published case reports. Bire et al. [12] studied the number of myxoma cases in patients over 75 years of age between 1962 and 1997 and found only 19 confirmed cases. The morphology and motility of myxoma and thrombus may be similar and difficult to distinguish by echocardiography, and surgical excision may be necessary for certain diagnosis [13]. Due to low morbidity and mortality besides its excellent outcome even in the elderly, surgery is the preferred choice of treatment for LAMs.

The main limitation of our work is the absence of Trans-oesophageal echo. (TOE) and histological confirmation of the diagnosis because the patient did not undergo TOE and surgical intervention; moreover we could perform neither CT nor MRI for a more precise definition of location and extension.

\section{Conclusion}

When clinical and echocardiographic features strongly support the diagnosis of myxoma (in term of location and echocardiographic appearance) additional imaging with CT and MRI may be unnecessary and reasonable diagnosis can be made even in the absence of histology based findings. Regarding treatment, conservative management is of limited value in symptomatic patients with large myxomas. However, a conservative strategy with echocardiographic monitoring, \& anticoagulation is favoured in high operative risk patients, asymptomatic patients, and slow growing atrial myxomas.

\section{References}

1. Hart RG, Albers G, Koudstaal P (1998) Cardioembolic stroke. Ginsberg M, Bogousslavsly J(Eds.), Cerebrovascular Disease: Pathophysiology, Diagnosis and Management. Blackwell Science; 1392-429.

2. Wessler BS, Kent DM (2015) Controversies in cardioembolic stroke. Curr Treat Options Cardiovasc Med 17: 358. [Crossref]

3. Arboix A, Alió J (2010) Cardioembolic stroke: clinical features, specific cardiac disorders and prognosis. Curr Cardiol Rev 6: 150-161. [Crossref] 
Paul GK (2016) A case report of recurrent syncope \& transient ischaemic attack in elderly man

4. Delewi R, Zijlstra F, Piek JJ (2012) Left ventricular thrombus formation after acute myocardial infarction. Heart 98: 1743-1749. [Crossref]
5. TunstallPedoe DS (2007) Marathon cardiac deaths: the london experience. Sports Med 37: 448-450. [Crossref]

6. Bolognesi M, Bolognesi D (2014) Asymptomatic Ischemic Heart Disease in a 45-yearold Male Athlete: A Case Report. J Gen Pract 2: 139.

Copyright: (C2016 Paul GK. This is an open-access article distributed under the terms of the Creative Commons Attribution License, which permits unrestricted use, distribution, and reproduction in any medium, provided the original author and source are credited. 